\title{
INFLUÊNCIA DA PRESSÃO TRANSMEMBRANAR EM UM REATOR DE MEMBRANA PARA PURIFICAÇÃO DO BIODIESEL
}

\author{
C. G. QUEIROZ ${ }^{1}$, E. A. SANTOS ${ }^{1}$, G. A. G. PEDROZA ${ }^{1}$, S. A. A. PAÍS ${ }^{1}$, I. T. SANTOS ${ }^{2}$, \\ D. F. ALMEIDA' ${ }^{2}$, T. C. B. CRUZ ${ }^{2}$, L. C. L. SANTOS ${ }^{3}$, A. K. C. L. LOBATO ${ }^{1}$ \\ ${ }^{1}$ Universidade Federal da Bahia, Departamento de Engenharia Química \\ ${ }^{2}$ Universidade Salvador, Departamento de Engenharia Química \\ ${ }^{3}$ Universidade Federal da Bahia, Departamento de Ciência e Tecnologia dos Materiais
}

E-mail para contato: katerine.carvalho@ufba.br

\begin{abstract}
RESUMO - O biodiesel é produzido através da reação de transesterificação, que consiste em uma reação química envolvendo um álcool e um triglicerídeo, formando ésteres de ácidos graxos e glicerol. A tecnologia de membrana tem sido explorada na separação dos produtos obtidos na reação de transesterificação, na qual possui uma barreira seletiva que regula o transporte de fluidos. Alguns fatores são determinantes para separação em processos que envolvem membranas, como seletividade, diâmetro de poro, pressão e temperatura. O presente trabalho tem como objetivo avaliar o efeito da pressão transmembranar na conversão do biodiesel e no fluxo do permeado, utilizando uma membrana cerâmica de $0,2 \mu \mathrm{m}$ de diâmetro de poro e variando a pressão no intervalo de $0,2-0,9 \mathrm{kgf} / \mathrm{cm}^{2}$. Os resultados mostraram que o fluxo do permeado sofre efeito com a elevação da pressão transmembranar, produzindo uma maior quantidade de glicerina, a medida que a pressão aumenta. $\mathrm{Na}$ análise da conversão da reação, notou-se que a pressão não interfere na qualidade do biodiesel, uma vez que toda a reação ocorre no reator de mistura, já alcançando valores máximos de conversão.
\end{abstract}

\section{INTRODUÇÃO}

Estudos no campo de ciências e tecnologias no âmbito de fontes renováveis de energia tem se tornado cada vez mais importante no cenário mundial, em função da escassez e dos altos impactos ambientais provocados pelos combustíveis fósseis (Montané et al.,2011). Umas das alternativas encontradas é a utilização do biodiesel, combustível limpo, biodegradável e não tóxico.

O biodiesel pode ser produzido utilizando óleos vegetais, gordura de origem animal ou óleos residuais. No Brasil a diversidade climática e o extenso território favorece o cultivo de matérias-primas para a produção de biodiesel (Sdrula, 2009). Dentre as oleaginosas utilizadas na produção de biodiesel pode-se citar soja, dendê, girassol, canola e pinhão manso (Koh e Ghazi, 2011). 
O processo empregado para converter matérias-primas graxas em biodiesel é chamado de transesterificação. Segundo Gomes et al. (2011), a transesterificação é uma reação química entre triglicerídeos e um álcool de cadeia curta, na presença de um catalisador ácido ou básico, formando glicerina, ésteres de cadeias longa de ácidos graxos e co-produtos.

Na separação dos produtos obtidos na reação de transesterificação, um dos métodos utilizados é a purificação via lavagem com água. Essa lavagem é usada para remover os remanescentes de catalisador, álcool e glicerina. No entanto, esse processo de purificação gera uma grande quantidade de efluentes a ser descartado acarretando, além de um impacto ambiental, um consumo excessivo de água (Atadashi et al., 2012). Nesse contexto, processos alternativos vêm sendo estudados para a purificação do biodiesel, a exemplo do reator de membrana.

Atualmente, a tecnologia de membrana tem sido explorada na purificação do biodiesel, na qual possui uma barreira seletiva que regula o transporte de fluidos. Nesse processo, a membrana atua removendo a glicerina da corrente do biodiesel ou retendo os triglicerídeos que não foram reagidos (Shuit et al., 2012). A utilização de membrana na área de biocombustíveis ainda é pouco estudada, porém promissora por apresentar vantagens na economia de energia e praticidade na separação do biodiesel.

Assim, o presente trabalho tem como objetivo avaliar o efeito da pressão transmembranar, utilizando uma membrana cerâmica de $0,2 \mu \mathrm{m}$ de diâmetro de poro. As análises foram identificadas através da verificação do fluxo do permeado e da qualidade do produto, tendo como referência, o fluxo de glicerina permeada e a conversão do biodiesel.

\section{MATERIAIS E MÉTODOS}

\subsection{Materiais}

Óleo de soja (marca Soya, fabricante Bunge Alimentos S.A, lote: 0311); Álcool metílico P.A. 99,8\% de pureza (fabricante: Anidrol Produtos para Laboratórios, lote: 24.998); Hidróxido de potássio P.A. (fabricante: Vetec Química Fina, lote: 1102011) e Heptano (mistura de isômeros) P.A. (fabricante: Química Moderna, lote: 00143).

\subsection{Planta piloto de produção de biodiesel}

A implantação de um sistema reacional utilizando membranas, para produção de biodiesel via rota metílica, a partir da reação de transesterificação do óleo de soja usando o mecanismo da catálise homogênea foi o objetivo geral da concepção da planta piloto. Entretanto, a construção do sistema permite a exploração de diversas linhas de pesquisa envolvendo a produção de biodiesel, como exemplo, a avaliação da influência da pressão transmembranar no volume do permeado produzido durante o processo e na conversão de ésteres metílicos. A Figura 1 esboça o fluxograma do processo estudado. 


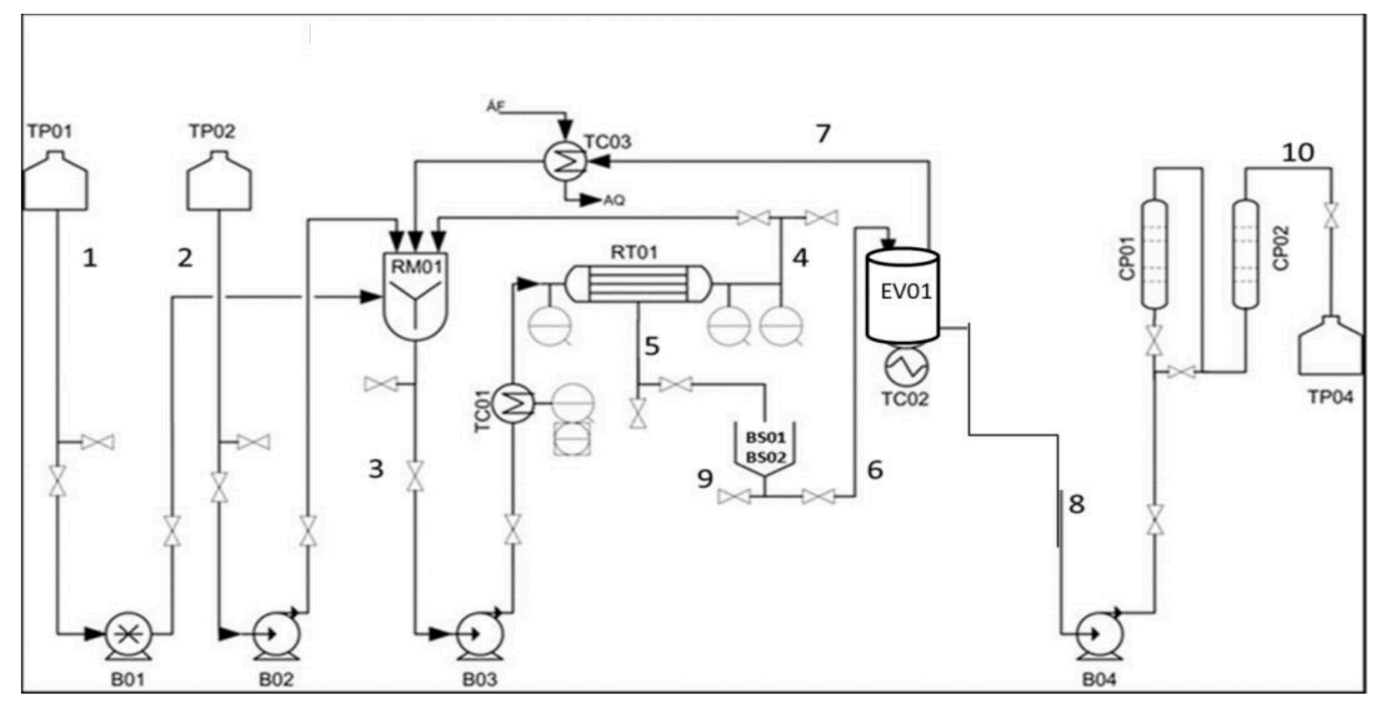

Figura 1 - Fluxograma do processo.

Legenda: $\mathrm{AF}=$ Água fria; $\mathrm{AQ}=$ Água quente; B01, B02, B03 e B04 = Bombas peristálticas; BS01 e BS02 = Decantadores; CP01 e CP02 = Colunas de purificação; EV01 = Evaporador 01; RM01 = Reator de mistura 01; RT01 = Reator de membrana tubular 01; TC01, TC02 e TC03 = Trocadores de calor; TP01, TP02 e TP04 = Tanques pulmão.

\subsection{Membrana cerâmica inerte}

Utilizou-se um sistema reacional em pequena escala com uma membrana cerâmica inerte Membralox, modelo: ET1-070, como diâmetro do poro de 0,2 $\mu \mathrm{m}$, diâmetro do canal de $7 \mathrm{~mm}$, comprimento de $250 \mathrm{~mm}$, área de filtração de $0,005 \mathrm{~m}^{2}$ e composição de $\alpha$ - alumina (fabricante ${ }^{\mathbb{C}}$ Pall Corporation).

\subsection{Procedimento experimental}

Foram adicionados aos TP01 e TP02 o óleo de soja (matéria-prima) e uma solução de metanol com $1 \%$ de $\mathrm{KOH}$ (catalisador homogêneo), respectivamente, seguindo uma razão estequiométrica de 9:1 metanol/óleo. Posteriormente as bombas B01 e B02 foram ligadas simultaneamente com vazões respeitando a razão estequiométrica utilizada para que o óleo e o metóxido (metanol $+\mathrm{KOH}$ ) chegassem juntos ao reator RM01 onde a mistura foi mantida por vinte minutos, sob agitação de $350 \mathrm{rpm}$ e a uma temperatura de $60^{\circ} \mathrm{C}$. Segundo Andrade (2013), essas condições de operação são consideradas mais favoráveis para uma melhor conversão do triacilglicerol em éster utilizando este sistema. Após o tempo de reação a mistura foi bombeada, através da bomba B03, levada para o trocador de calor, TC01, e em seguida para a membrana cerâmica inerte, RT01, na qual ocorre a permeação dos produtos (biodiesel e glicerina), como também dos reagentes não convertidos (mono, diglicerídeos e álcool) e do catalisador. O que não foi permeado pela membrana retorna ao reator RM01, por reciclo. A retirada das amostras do permeado foram feitas através dos decantadores BS01 e BS02. 
Para a análise da pressão transmembranar, foram avaliados a conversão do biodiesel através dos índices de mono, di e triglicerídeos das amostras por cromatografia gasosa, utilizando um cromatógrafo modelo HP-5890, seguindo a norma ASTM D6584 adaptada. No sistema, foram aplicadas pressões variando de 0,2 a $0,9 \mathrm{kgf} / \mathrm{cm}^{2}$, com intervalos de 0,1 $\mathrm{kgf} / \mathrm{cm}^{2}$ para a avaliação da conversão e com intervalos de $0,2 \mathrm{kgf} / \mathrm{cm}^{2}$ para a verificação do fluxo do permeado. O controle da pressão transmembranar foi realizado de forma manual por uma válvula agulha Hoke ${ }^{\mathbb{O}}$ em aço inox e um manômetro digital $\mathrm{ABC}$ instrumentação (0-1,6 $\mathrm{kgf} / \mathrm{cm}^{2}$ ). Em cada pressão estudada, a coleta do volume do permeado foi realizada ao longo de 40 minutos. Assim, foi possível o cálculo do fluxo de biodiesel e glicerina que foi permeado, através da Equação 1:

$$
J_{P e r m}=\frac{V_{P}}{A \cdot t}
$$

onde $\mathrm{J}_{\text {Perm }}$ é o fluxo do permeado $\left(\mathrm{mL} \mathrm{m}^{-2} \min ^{-1}\right), \mathrm{V}_{\mathrm{p}}$ é o volume do permeado $(\mathrm{mL})$, A é a área de permeação da membrana $\left(\mathrm{m}^{2}\right)$ e $\mathrm{t}$ é o tempo (minutos).

Para as análises de conversão da reação, avaliou-se apenas os 10 primeiros minutos, considerando que após esse período toda a reação já ocorreu e a conversão já alcançou o valor máximo. As análises cromatográficas foram realizadas com as amostras contendo $0,1 \mathrm{~g}$ do permeado e $10 \mathrm{~mL}$ de heptano (que funciona como solvente de diluição).

\section{RESULTADOS E DISCUSSÕES}

\subsection{Análise da conversão da reação}

A conversão encontrada em cada pressão é reportada na Figura 2. Os valores de conversão não ultrapassam $99 \%$ em função de alguns componentes do óleo vegetal não serem saponificáveis. Isso significa que alguns traços desses materiais além de possuírem um baixo grau de volatilidade, são considerados estáveis em bases. No entanto, pode-se considerar vantajoso o fato dessas substâncias não reativas serem oleofílicas e assim sendo, ficam retidas na membrana com os triglicerídeos. 


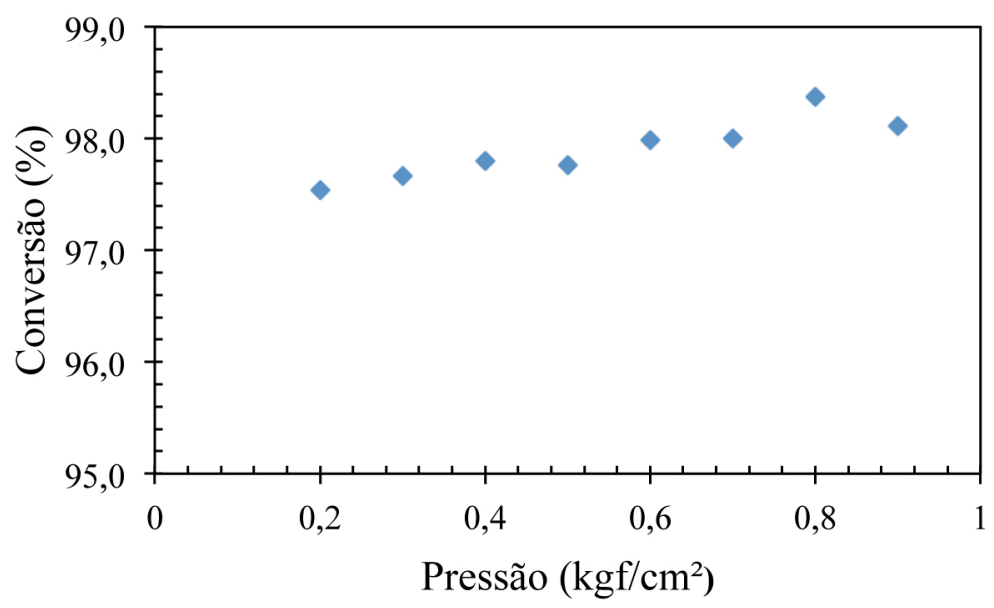

Figura 2 - Conversão em função da pressão de operação.

Como pode ser visto na Figura 2, em todas as pressões foram alcançados altos níveis de conversão, de modo que verifica-se que não há uma variação significativa na conversão entre as pressões de operação utilizadas.

A principal razão para obtenção de altas conversões utilizando o sistema com membrana é a redução da quantidade de triglicerídeos no produto final e uma maior concentração retida na membrana. A pressão transmembranar não influencia na conversão da reação em função de que toda a reação ocorre no reator de mistura, à montante da membrana, alcançando seus valores máximos antes da etapa de purificação.

Nas Figuras 3 e 4, são mostradas, respectivamente, concentração de mono e diglicerídeos, presentes no permeado, em função do tempo para diferentes pressões. Traços de monoglicerídeos aparecem em todas as corridas para todas as pressões ao passo que diglicerídeos possui menores concentrações em tempos maiores e pressões mais elevadas. Alguns desníveis no gráfico podem ocorrer em função das reações serem reversíveis e assim, o equilíbrio se desloca constantemente. 


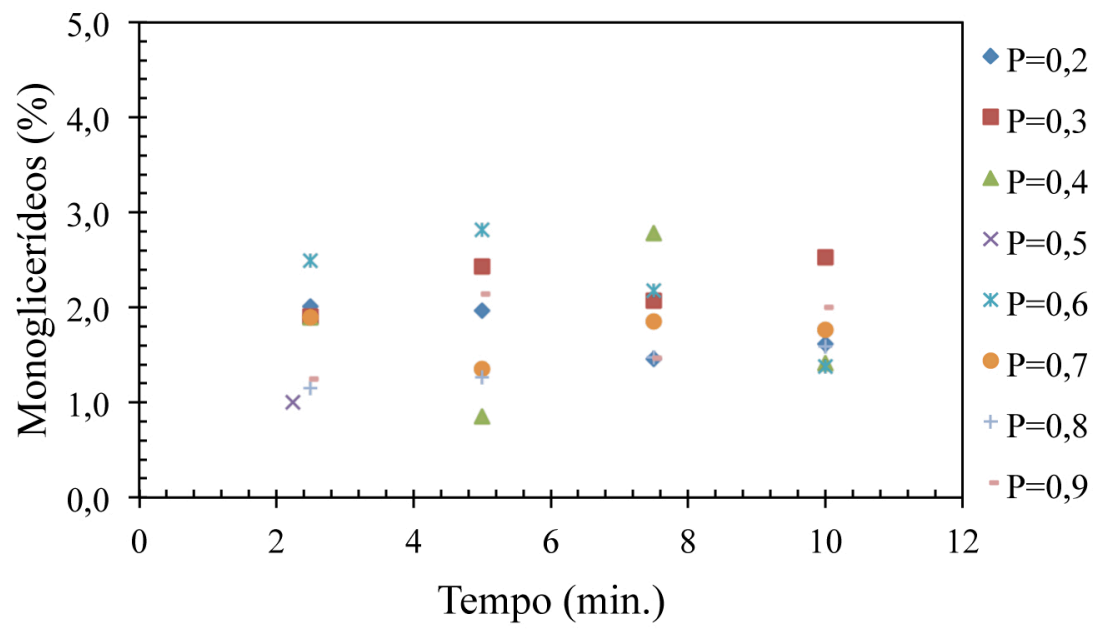

Figura 3 - Concentração de monoglicerídeos em função do tempo para diferentes pressões $\left(\mathrm{kgf} / \mathrm{cm}^{2}\right)$.

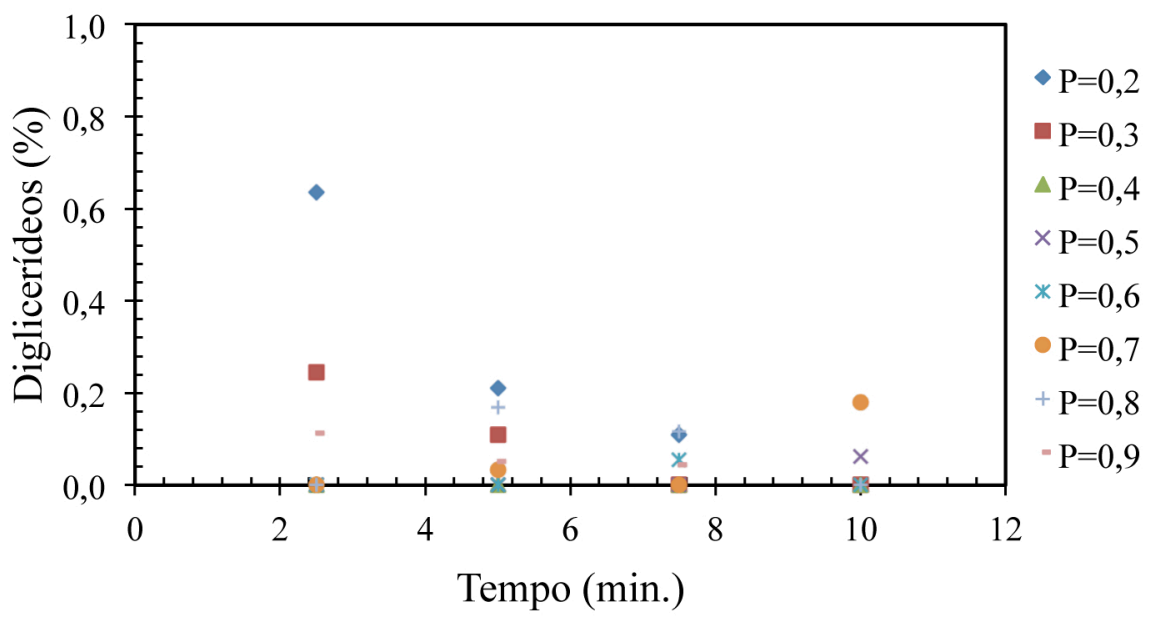

Figura 4 - Concentração de diglicerídeos em função do tempo para diferentes pressões $\left(\mathrm{kgf} / \mathrm{cm}^{2}\right)$.

As concentrações de triglicerídeos não foram representadas graficamente em função de que não houve valores significativos dessas moléculas na corrente do permeado. Isso ocorre devido ao papel da membrana em reter os triglicerídeos em função do maior tamanho de suas moléculas.

Os mono e diglicerídeos são produtos intermediários da reação de transesterificação e podem permanecer na fase óleo, formar micelas ou se solubilizarem no metanol na fase rica em biodiesel. Di e triglicerídeos não são espontaneamente miscíveis no metanol ao passo que o monoglicerídeo se mistura com mais facilidade (CAO et al, 2007). Dessa maneira, espera-se que os triglicerídeos fiquem retidos na membrana, os diglicerídeos permaneçam parcialmente retidos e o monoglicerídeo apareça em maior quantidade no permeado. 


\subsection{Análise do fluxo do permeado}

Na Figura 5, é apresentado o fluxo de biodiesel e glicerina em função da pressão, onde nota-se que com o aumento da pressão transmembranar, o fluxo do permeado também aumenta. Segundo Shuit et al. (2012) e Atadashi et al. (2012), isso ocorre em função de uma maior força de condução aplicada para a separação. Em maiores pressões, a força matriz das moléculas é aumentada, resultando em taxas de transferências de massa mais elevadas.

Além disso, a pressão transmembranar está correlacionada com a viscosidade da mistura reacional. Com uma maior pressão, há um maior teor de glicerina presente no permeado. A solubilidade da glicerina e metanol e a solubilidade do biodiesel e metanol resulta na dissolução de glicerina no biodiesel, facilitando a passagem de glicerina pelo permeado (Saleh et al., 2010; Shuit et al., 2012). Em função disso, na pressão de 0,2 $\mathrm{kgf} / \mathrm{cm}^{2}$, não houve produção de glicerina no permeado ao passo que a medida que a pressão foi aumentando, o fluxo de glicerina no permeado seguiu a mesma tendência.

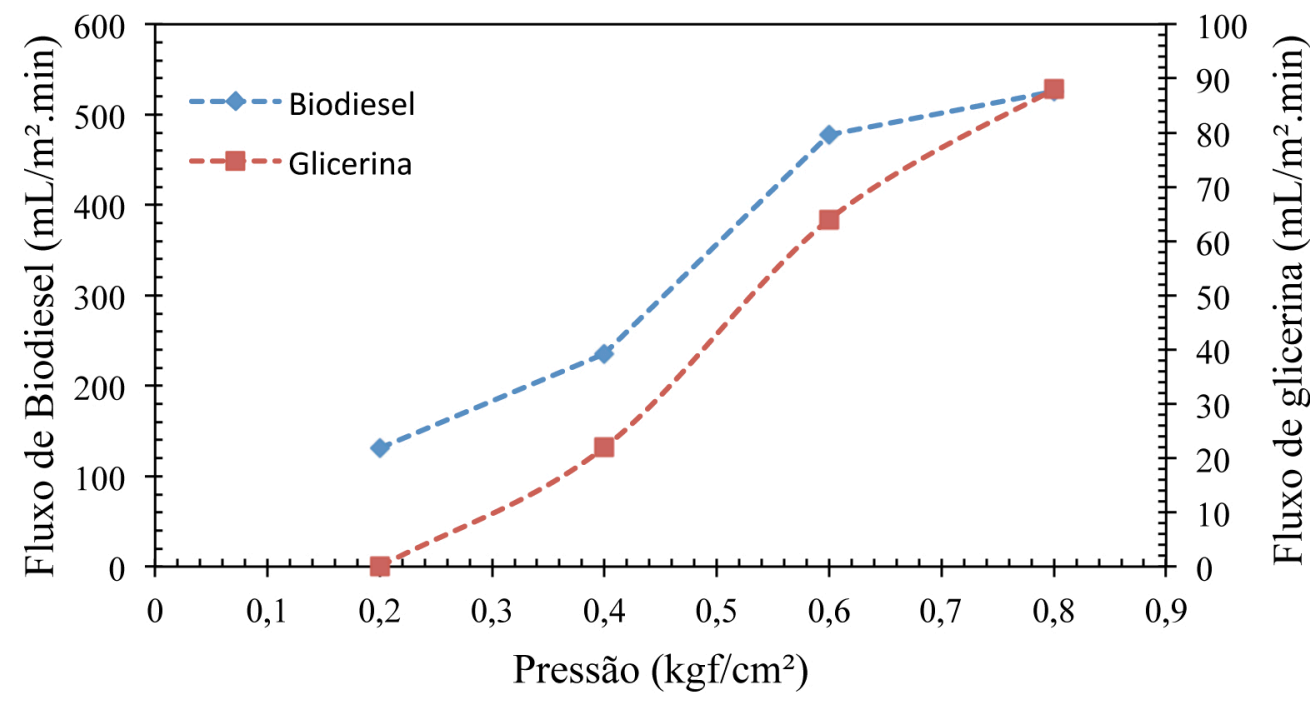

Figura 5 - Fluxo de biodiesel e glicerina em função da pressão de operação.

\section{CONCLUSÕES}

A conversão da reação foi analisada e constatou-se que, nas condições estudadas, a pressão transmembranar não tem influência na qualidade do biodiesel produzido, em termos de conversão. Foram verificadas as concentrações de mono, di e triglicerídeos, e notou-se que os triglicerídeos ficaram retidos na membrana obtendo altos resultados de conversão, independente da pressão transmembranar submetida ao sistema. Além disso, percebeu-se que pressões elevadas implicam num maior fluxo do permeado e consequentemente uma maior quantidade de glicerina produzida. Já na pressão de $0,2 \mathrm{kgf} / \mathrm{cm}^{2}$, não houve permeação de glicerina. Traços de catalisador que porventura possam estar presentes no biodiesel poderão ser removidos facilmente através de resinas de troca iônica. Desta forma, em caso de 
ampliação de escala, talvez seja interessante trabalhar com um sistema de reatores de membranas ligados em série, onde o primeiro reator operando com altas pressões, elevando o fluxo do permeado, e o segundo reator operando com baixas pressões, separando o biodiesel da glicerina.

\section{AGRADECIMENTOS}

Os autores agradecem ao $\mathrm{CNPq}$ pelo financiamento do projeto 558847/2010-2, à CAPES e FAPESB pela concessão de bolsas, à UNIFACS e UESC pela parceria do projeto.

\section{REFERÊNCIAS}

ANDRADE, S., S., L. Avaliação da Produção de Biodiesel em Reator de Membrana. 2013. 118f. Dissertação (Mestrado em Engenharia Química) - Programa de Pós-Graduação em Engenharia Química da Universidade Federal da Bahia, Salvador, 2013.

ATADASHI, I.M.; AROUA, M.K.; AZIZ, A.R.A.; SULAIMAN, N.M.N. High quality obtained through membrane technology. Journal of Membrane Science, v.421-422, p.154$164,2012$.

BATTISTI, A.P.; ELIAS, D.T.; DIAS, R.; QUADRI, M.B. Determinação da conversão do óleo em biodiesel por RMN ${ }^{1} \mathrm{H}$ e HPLC na reação de transesterificação. In: VIII Congresso Brasileiro de Engenharia Química em Iniciação Científica. Anais.Uberlândia (MG): UFSC, 2009.

GOMES, M.C.S.; FERREIRA, M.Z.; TONELLI, V.P.; BARROS, S.T.D. de; PEREIRA, N.C. Aplicação de microfiltração com membranas cerâmicas no processo de separação de biodiesel e glicerina. Acta Scientiarium. Tecnology, v.33, p.415-419, 2011.

KOH, M.Y.; GHAZI, T.I.M. A review of biodiesel production from Jatropha Curcas L. oil. Renewable and Sustainable Energy Reviews, v.15, p. 2240-2251, 2011.

MONTANÉ, D.; BOLSHAK, E.; ABELLÓ, S. Thermodynamic analysis of fuel processors based on catalytic-wall reactors and membrane systems for ethanol steam reforming. Chemical Engineering Journal, v.175, p.519-533, 2011.

SALEH, J.; DUBÉ, M. A.; TREMBLAY, A.Y. Effect of Soap, Methanol, and Water on Glycerol Particle in Biodiesel Purification. Energy Fuels, v.30, p.6179-6186, 2010.

SDRULA, N. A study using classical or membrane separation in the biodiesel process. Desalination, v.250, p.1070-1072, 2009.

SHUIT, S.H.; ONG, Y.T.; LEE, K.T.; SUBHASH, B.; TAN, S.H. Membrane technology as a promising alternative in biodiesel production: a review. Biotechnology Advances, v.30, p.1364-1380, 2012. 